\title{
Guidance Law Design for Terminal Area Energy Management of Reusable Launch Vehicle by Energy-to-Range Ratio
}

\author{
Wen Jiang and Zhaohua Yang \\ School of Instrumentation Science and Optoelectronics Engineering, Beihang University, Beijing 100191, China \\ Correspondence should be addressed to Wen Jiang; jiangwen-1937@163.com
}

Received 17 February 2014; Accepted 15 April 2014; Published 12 May 2014

Academic Editor: Weichao Sun

Copyright (c) $2014 \mathrm{~W}$. Jiang and Z. Yang. This is an open access article distributed under the Creative Commons Attribution License, which permits unrestricted use, distribution, and reproduction in any medium, provided the original work is properly cited.

\begin{abstract}
A new guidance scheme that utilizes a trajectory planning algorithm by energy-to-range ratio has been developed under the circumstance of surplus energy for the terminal area energy management phase of a reusable launch vehicle. The trajectory planning scheme estimates the reference flight profile by piecing together several flight phases that are defined by a set of geometric parameters. Guidance commands are readily available once the best reference trajectory is determined. The trajectory planning algorithm based on energy-to-range ratio is able to quickly generate new reference profiles for testing cases with large variations in initial vehicle condition and energy. The designed flight track has only one turn heading, which simplifies the trajectory planning algorithm. The effectiveness of the trajectory planning algorithm is demonstrated by simulations, which shows that the guided vehicle is able to successfully dissipate energy and reach the desired approach and landing glideslope target with small tracking errors.
\end{abstract}

\section{Introduction}

The descent flight of the reusable launch vehicle (RLV) commonly comprises three mission phases: the reentry phase, the terminal area energy management (TAEM) phase, and the approach and landing phase. The TAEM phase follows the reentry phase and precedes the approach and landing phase. A major breakthrough of the guidance design of TAEM vehicles can be attributed to the space shuttle entry guidance [1]. The TAEM phase is in charge of aligning the vehicle with the runway while managing the vehicle's kinematic and potential energies. In other words, at the end of TAEM phase, the total energy of the vehicle should be consistent with a safe landing.

The only TAEM guidance strategy fully operational currently follows one of NASA's space shuttles. The trajectory is composed of four different branches: energy dissipation Sturn phase, acquisition phase, heading alignment phase, and prefinal phase. The start of the TAEM phase is commonly referred to as the terminal entry point (TEP) and its end as the approach and landing interface (ALI). TEP and ALI are symbolized by altitude and Mach number. Usually, typical altitude and Mach number values at TEP and ALI are given.
For the US space shuttle, TAEM begins at approximately Mach 2 and ALI is nominally at 10,000 ft altitude and Mach 0.5 [2]. In earlier version, the space shuttle's TAEM guidance scheme is based on four heading alignment cylinders (HAC) while the recent version is based on cones. Depending on the vehicle's energy at TEP, the guider can choose HAC to fly.

In many missions, the ultimate aim is to recover the vehicle on a runway through an appropriate TAEM guidance, followed by appropriate landing logic through typical glide slope and flare [3]. In [4], a methodology to obtain vehicle performance, pneumatic coefficients, and couple it to a code that computes reentry trajectories considering six degrees of freedom was proposed. There have been some attempts in the recent literature to generate feasible TAEM trajectories. Horneman and Kluever [5] have presented a TAEM guidance methodology that employs a trajectory planning algorithm that computes a feasible path from the current state to the desired ALI state without relying on a precomputed, stored database of neighboring TAEM trajectories. Kluever et al. [6] have proposed an algorithm capable of rapidly generating a feasible TAEM path from the current state to the desired ALI state. Their path-planning algorithm adjusts the TAEM trajectory by iterating on two geometric parameters: 
the down-track location of the HAC and its radius. Filipe and Weerdt [7] have used an interval analysis to optimize the trajectory of an RLV during the TAEM phase. The algorithm presented by Filipe et al. [7] is improved in terms of rigor and efficiency.

Future RLV may greatly benefit from advanced guidance and control technologies. Abbasi and Mortazavi [8] have presented a new concept for atmospheric reentry online optimal guidance and control using a method called matched asympotic reentry guidance and control (MARE G\&C) that exploits the different time scale featured by reentry dynamics. Hanson [9-11] has argued that advanced guidance and control will greatly improve safety and reliability by successfully returning an RLV that is plagued by aerosurface failures, poor vehicle performance, and larger-than-expected flight dispersions. Recently, abundant work have applied advanced guidance concepts to the TAEM phases of an RLV. Kluever [12] has presented a TAEM guidance method for a scenario where the banking capabilities of the RLV are compromised. The proposed TAEM guidance algorithm computes the best feasible path from the current state to the desired ALI state by iterating on two guidance parameters and numerically propagating several trajectories. Burchett [13] developed a TAEM guidance method based on fuzzy logic which is capable of handling trajectory constraints. Grantham [14] presented a guidance method based on an adaptive critic neural network, and this work presents trajectories with limited heading changes. Hall and Shtessel [15] presented the first application of sliding mode disturbance observer driven sliding mode control to improve RLV flight control performance in TAEM.

The RLV approach has been proved to be robust to uncertainties in many factors and not to take full advantage of the vehicle's capabilities. Many guidance schemes have been proposed to overcome the same problem. Aiming at surplus energy at TEP, in this paper, a new method during the TAEM phase based on energy-to-range ratio (TAEM E-R) is used to plan the trajectory of the RLV.

In Section 2, the equations of motion modeling the movement of an RLV during the TAEM phase will be quickly derived. Moreover, the underlying assumptions will be pointed out and the atmospheric and vehicle models specified. The trajectory design methodologies and the virtues of this mode will be described, respectively, in Section 3. In Section 4, results and analysis of simulation will be presented. Conclusions and suggestions for future work will be given in Section 5 .

\section{Equations of Motion for the RLV during TAEM Phase}

This section is dedicated to the derivation of the equations of motion for an RLV during the TAEM phase. These equations are constructed on a set of assumptions as follows.

(1) The RLV is assumed to be a rigid body; the elastic deformation is ignored.

(2) The gravity acceleration is assumed to be constant.
(3) The Earth is assumed to be flat, nonrotating, and inertial.

(4) All the turns are assumed to be coordinated turns, and there are no aerodynamic side forces since the sideslip angle is assumed to be zero.

2.1. The Equations of Motion. The appropriate governing equations of motion for the vehicle during TAEM phase are

$$
\begin{aligned}
& \dot{V}=-\frac{D}{m}-g \sin \gamma, \\
& \dot{\gamma}=\frac{L \cos \mu}{m V}-\frac{g \cos \gamma}{V}, \\
& \dot{\chi}=\frac{L \sin \mu}{m V \cos \gamma}, \\
& \dot{h}=V \sin \gamma, \\
& \dot{x}=V \cos \gamma \cos \chi, \\
& \dot{y}=V \cos \gamma \sin \chi,
\end{aligned}
$$

where $V$ is the velocity, $m$ is the mass of the RLV, $g$ is the acceleration due to gravity, $\gamma$ is the flight path angle, $\chi$ is the vehicle's heading angle relative to the runway's centerline, $\mu$ is the bank angle about the velocity vector, $h$ represents the altitude, and $x$ and $y$ are the horizontal positional coordinates of the RLV; the $+x$ axis points along the run way centerline on approach and the $+y$ axis points right of the runway on approach.

2.2. Atmospheric Model. To reduce the computational complexity, the simple and time-invariant exponential atmospheric model is used to simulate the atmosphere. The exponential model assumes that the air density $\rho$ decreases exponentially with increasing altitude $h$ as follows:

$$
\rho(h)=\rho_{0} \exp \left(-\frac{h}{H}\right),
$$

where $\rho_{0}=1.752 \mathrm{~kg} / \mathrm{m}^{3}$ is the air density at the reference altitude $(h=0) . H$ is the scale height which equals $7249 \mathrm{~m}$.

2.3. Vehicle Model. The simplified aerodynamic model was used in the formulation as follows:

$$
\begin{gathered}
D=q S C_{D}, \quad C_{D}=C_{D}(\alpha, M), \\
L=q S C_{L}, \quad C_{L}=C_{L}(\alpha, M), \\
q=\frac{1}{2} \rho(h) V^{2},
\end{gathered}
$$

where $D$ is the aerodynamic drag force, $L$ is the aerodynamic lift force, $q$ is the dynamic pressure, and $S$ is the reference area of the RLV. The aerodynamic drag coefficient $C_{D}$ and lift coefficient $C_{L}$ are assumed to be dependent only on angle of attack $\alpha$ and Mach number $M$, an assumption employed in other publications related to the TAEM phase. 


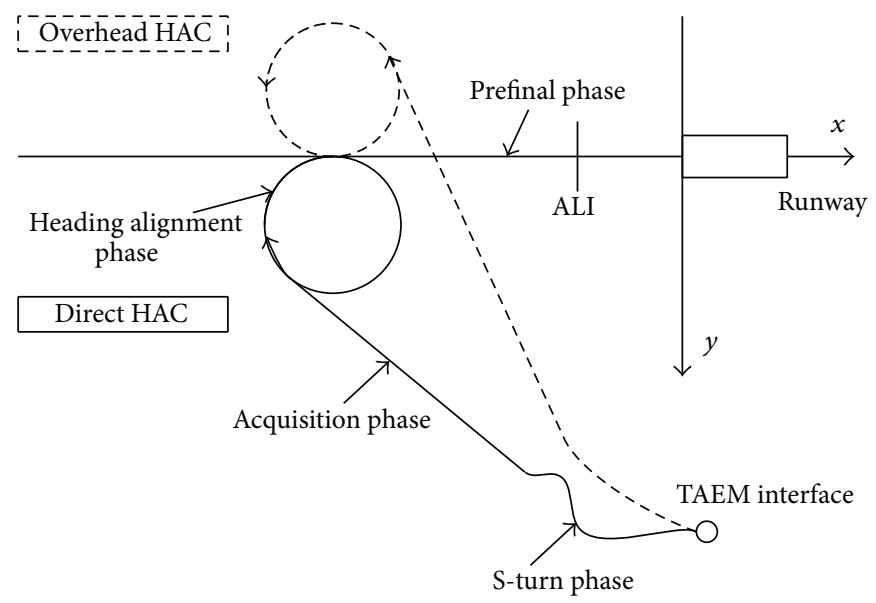

FIgURE 1: TAEM guidance phases and approaching method (solid line: direct HAC; dashed line: overhead HAC).

\section{TAEM Trajectory Planning Algorithm}

The TAEM guidance strategy fully operational currently is in accordance with space shuttle. The various flight segments of the reference TAEM ground track are plotted in Figure 1. In the energy dissipation S-turn phase, TAEM begins with the S-turn phase to dissipate surplus energy by changing course and increasing flight range. It is not the integrant part of TAEM phase except energy excess at TEP. During the acquisition phase, the vehicle is banked until it can align its heading with a tangency point on the preplanned HAC and fly a straight-line path to the point. At the tangency point of the heading aligning phase, the RLV banks and turns to follow the HAC, where the ground track is an inward spiral, until the vehicle can align the runway centerline. When the HAC turn is completed, the vehicle flies the prefinal phase, which is a straight-line path along the runway centerline to the ALI. Regulating energy to make the flight states (altitude, velocity, and position) of RLV can meet the requirements of unpowered autolanding.

In a classical guidance scheme, TAEM guidance may use a direct HAC mode, or an overhead HAC mode. A direct $\mathrm{HAC}$ is located on the same side of the runway, and the corresponding HAC turn angle is less than 180 degrees (solid line in Figure 1). An overhead HAC is on the opposite side of the runway, and the ensuing turn angle is greater than 180 degrees (dashed line in Figure 1). After the HAC turn is completed, the prefinal TAEM phase is initiated, and the RLV follows a straight-line ground track to ALI.

In the situation of energy surplus, the approaching method (Figure 2) proposed in this paper is different from the classical mode. In acquisition phase, the RLV turns to align its heading, flies a straight-line path to the circumcircle tangency point of two virtual HACs and turns to follow the circumcircle not the HAC immediately, and then follows the opposite HAC to the prefinal phase. The characteristics of this new mode are as follows.

(1) The selection of approaching mode is unnecessary, which simplifies the trajectory planning algorithm. The designed flight track has only one turn heading

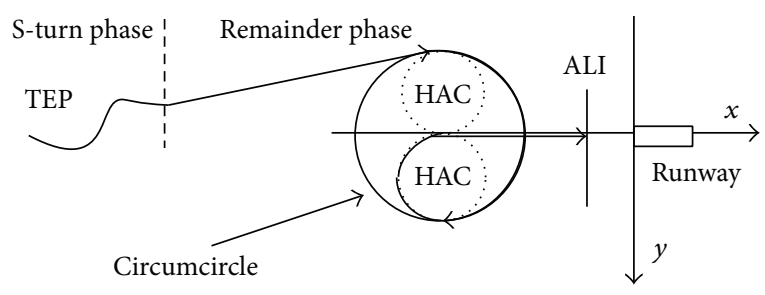

Figure 2: Approaching method based on TAEM E-R for energy surplus proposed in this paper.

by selecting an initial tangent line and a HAC after the initial conditions are determined to guarantee the continuity of heading angle and the ease of calculation.

(2) Initial surplus energy which varies extensively is allowable, for the range will adjust with the energy of TEP and ALI. The radius of HAC can be adjusted slightly after the S-turn phase is finished which will have no influence on the other guidance algorithm.

TAEM trajectory planning scheme adopts the E-R to divide the TAEM phase into S-turn phase and remainder phase. The S-turn phase terminates while the ratio $K_{\mathrm{el}}$ decreases to a certain numerical value $K_{s}$. This value is determined by the dissipative energy of drag work in the remainder phase. The RLV flies to follow the remainder phase subsequently. In classical guidance scheme, both overhead HAC mode and direct $\mathrm{HAC}$ mode, many possibilities exist in drawing a tangent line to HAC. In this paper, designing the circumcircle of HAC is adopted. The center of the circle is in the horizontal axis, and at the beginning of acquisition phase, the vehicle banks to follow the tangent line to the circumcircle and then turns to follow the circular arc to HAC. After the initial conditions are given, we can confirm tangent direction firstly and design the tangent line, which has the only heading in each point, and compute remainder E-R in each point. If the ratio does not reach the certain value $K_{s}$, the vehicle keeps on $S$-turn flight to dissipate more energy until the ratio descends 


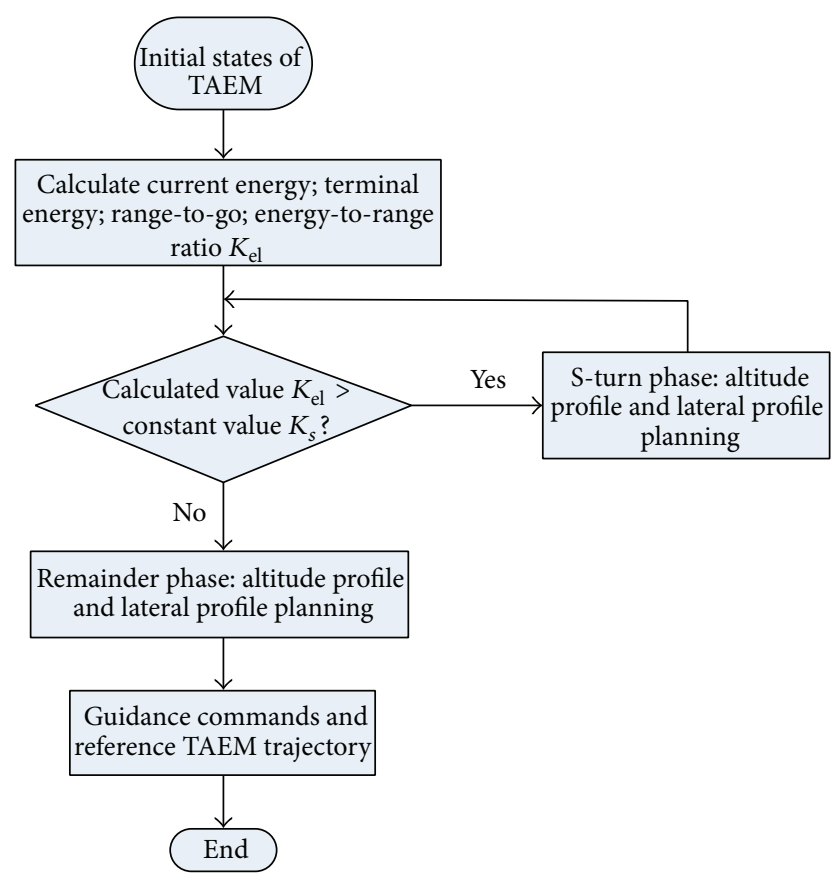

FIgURE 3: Flowchart of the TAEM E-R trajectory planning algorithm.

to the certain value $K_{s}$. Figure 3 presents the detailed logic of the TAEM trajectory planning algorithm.

3.1. Ground Track Range Prediction. The mechanical energy of unpowered RLV is defined in this paper as

$$
E=\frac{m V^{2}}{2}+m g h .
$$

The normalized total energy of the vehicle is defined as

$$
\frac{E}{m}=\frac{V^{2}}{2}+g h .
$$

Normalized energy has the units of $\mathrm{J} / \mathrm{kg}$. The derivative of energy to ground track is

$$
\frac{d E}{d s}=-\frac{D}{\cos \gamma}=\frac{-C_{D} q S}{\cos \gamma}
$$

From (6), it can be seen that many methods such as increasing range, increasing dynamic pressure, and adjusting drag coefficient could be adopted to dissipate surplus energy. The objective of S-turn phase is to consume energy by increasing flight range.

An essential part of the TAEM guidance is the accurate prediction of the remaining ground track range to ALI. Total range-to-go at the initiation of TAEM is the sum of four geometric segments:

$$
s_{\mathrm{go}}=s_{1}+s_{2}+s_{3}+s_{4} \text {. }
$$

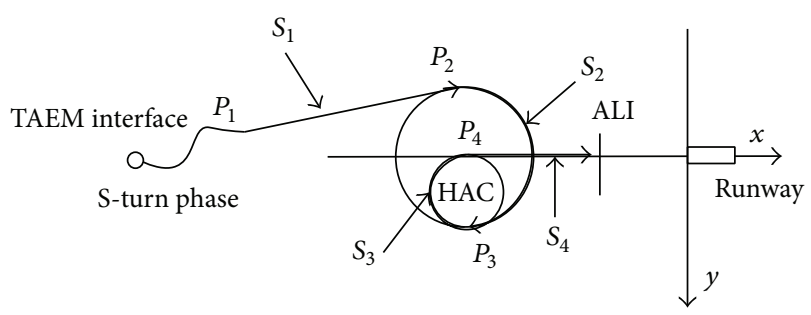

FIGURE 4: TAEM reference trajectory calculation.

As shown in Figure 4, the ground track range for the acquisition phase $s_{1}$ is equal to the length of tangent line $\mathrm{P}_{1} \mathrm{P}_{2}$, or the distance between current point and tangent point:

$$
s_{1}=\sqrt{\left(x-x_{\mathrm{P} 2}\right)^{2}+\left(y-y_{\mathrm{P} 2}\right)^{2}} \text {. }
$$

The ground track range for the heading align phase consists of $s_{2}$ and $s_{3} \cdot s_{2}$ represents arc length (i.e., $\mathrm{P}_{2} \mathrm{P}_{3}$ ) of the circumcircle of HAC,

$$
s_{2}=R \cdot \tau
$$

where $R$ represents radius of the circumcircle and $\tau$ is the arc angle. $s_{3}$ is half the circumference of HAC (i.e., $\mathrm{P}_{3} \mathrm{P}_{4}$ ),

$$
s_{3}=\pi \cdot R_{\mathrm{HAC}},
$$

where $R_{\text {HAC }}$ is the radius of HAC and $R=2 R_{\text {HAC }}$. Finally, the prefinal ground track range is simply the downrange distance from the HAC center to the ALI point,

$$
s_{4}=x_{\mathrm{ALI}}-x_{\mathrm{HAC}} \cdot
$$

In summary, the ground track segments can be computed from (7)-(11).

3.2. Reference Altitude Profile. The altitude profile planning is divided into two parts: S-turn phase and remainder phase.

To dissipate energy efficiently, the dynamic pressure maintains the maximum value during the S-turn phase:

$$
q_{\max }=\frac{1}{2} \rho v_{\max }^{2}
$$

The altitude of the S-turn phase $h_{S}$ has a direct relation to the air density, the corresponding altitude can be computed from (12), and the relevant velocity is given:

$$
h_{S}=-H \ln \left(\frac{2 q_{\max }}{\rho_{0} V^{2}}\right) .
$$

A polynomial defines the reference altitude profile of remainder phase, including acquisition phase, HAC heading alignment phase, and prefinal phase:

$$
h_{r}=c_{0}+c_{1} s+c_{2} s^{3 / 2}+c_{3} s^{2} .
$$

In (14), the independent variable $s$ is the remainder range of ground track and it makes the flight-path angle 
a continuous variable. $c_{0}, c_{1}, c_{2}, c_{3}$ are four undetermined polynomial coefficients. The restraints of initial altitude $h_{1}$, terminal altitude $h_{4}$, initial flight path angle $\gamma_{1}$, and terminal flight path angle $\gamma_{4}$ are described as

$$
\begin{aligned}
& h_{1}=c_{0}, \\
& h_{4}=c_{0}+c_{1} s+c_{2} s^{3 / 2}+c_{3} s^{2}, \\
& \frac{d h_{1}}{d s}=\tan \gamma_{1}=c_{1}, \\
& \frac{d h_{4}}{d s}=\tan \gamma_{4}=c_{1}+\frac{3}{2} c_{2} s^{1 / 2}+2 c_{3} s .
\end{aligned}
$$

The polynomial coefficients $\left(c_{0}, c_{1}, c_{2}, c_{3}\right)$ can be calculated from (15). Compared with the unaltered altitude profile that the space shuttle adopted, the reference altitude profile proposed in this paper preferably satisfies the needs of initial requirements such as altitude and flight-path angle for TAEM phase.

3.3. TAEM Guidance Law. S-turn phase adopts an open loop control mode in which the bank angle is fixed. With course changing, the flight range will increase. Since the overload and the pneumatic coefficient are restricted, the lift force, the flight radius per moment, and the variation of the heading angle are restricted. The certain bank angle, which has invariant absolute value, will change its direction by the given boundary. The boundary follows two rules simultaneously; firstly, the width of the boundary must guarantee that the vehicle arrives at the predetermined TAEM terminal point successfully; secondly, the times of bank angle reversal must be as few as possible.

The normative flight-path angle of the S-turn phase $\gamma_{s}$ is related to altitude and velocity; assume $k_{1}=d h / d V$; then

$$
\begin{aligned}
& \gamma_{s}=-\arcsin \frac{k_{1} D}{m\left(V+k_{1} g\right)}, \\
& L_{s}=\frac{\dot{\gamma} m V+m g \cos \gamma}{\cos \mu_{0}},
\end{aligned}
$$

where $L_{s}$ represents the lift force of the S-turn phase and bank angle $\mu_{0}$ is determinate, and in order to control the flight-path angle and the heading angle effectively, the value of the bank angle must be neither too large nor too small. Two boundaries were determinate according to the E-R:

$$
K_{\mathrm{el}}=\frac{\Delta E}{\Delta s},
$$

where $\Delta E$ is the surplus energy which will be dissipated. It can be computed by the present energy and ultimate energy. $\Delta s$ is the surplus ground track.

In the remainder phase, to ensure the restriction of dynamic pressure, the dynamic pressure $q_{r}$ is planned as

$$
q_{r}=q_{1}-\frac{q_{1}-q_{4}}{s_{\mathrm{go}}} s,
$$

where $q_{1}$ is the dynamic pressure of starting point of the acquisition phase. $q_{4}$ represents the dynamic pressure of ALI point. The velocity of TAEM in remainder phase can be computed from

$$
V_{r}=\sqrt{\frac{2 q_{r}}{\rho\left(h_{r}\right)}} .
$$

The derivative of altitude with respect to ground track is the tangent of the flight-path angle,

$$
\begin{aligned}
& \gamma_{r}=\arctan \left(c_{1}+\frac{3}{2} c_{2} s^{1 / 2}+2 c_{3} s\right), \\
& \mu_{r}=a \tan \left(\frac{\left(-\dot{\chi} m V_{r} \cos \gamma_{r}\right)}{\left(m V_{r} \dot{\gamma}_{r}+m g \cos \gamma_{r}\right)}\right) .
\end{aligned}
$$

Using the bank angle $\mu_{r}$ of (21), the lift force and the drag force will be calculated as follows:

$$
\begin{gathered}
\mu_{r} \neq 0 \quad L_{r}=\frac{-\dot{\chi} m V_{r} \cos \gamma_{r}}{\sin \mu_{r}}, \\
\mu_{r}=0 \quad L_{r}=\dot{\gamma}_{r} m V_{r}+m g \cos \gamma_{r}, \\
D_{r}=m g \sin \gamma_{r}+m \dot{V}_{r} .
\end{gathered}
$$

\section{Results for Trajectory Planning Algorithm and TAEM Simulation}

A baseline TAEM trajectory is obtained by executing the trajectory planning algorithm with a nominal entry interface state, a nominal ALI state, and nominal RLV aerodynamics. The initial TAEM state is taken from the end state of a nominal entry trajectory, with $h_{0}=28 \mathrm{~km}, V_{0}=1200 \mathrm{~m} / \mathrm{s}$, $\gamma_{0}=-3.4^{\circ}, \chi_{0}=-25^{\circ}, x_{0}=-60000 \mathrm{~m}$, and $y_{0}=-12000 \mathrm{~m}$. The nominal ALI state is $h_{\mathrm{ALI}}=3 \mathrm{~km}, V_{\mathrm{ALI}}=180 \mathrm{~m} / \mathrm{s}$, $\gamma_{\mathrm{ALI}}=-36^{\circ}, \chi_{\mathrm{ALI}}=0^{\circ}, x_{\mathrm{ALI}}=-8000 \mathrm{~m}$, and $y_{\mathrm{ALI}}=0 \mathrm{~m}$, respectively.

Results for the simulations are presented below. Figure 5 presents the altitude, velocity, energy, and dynamic pressure profile of the TAEM phase and Figure 6 plots the ground track trajectory. As expected, the parameter profiles and ground track show that the vehicle performs a feasible and successful flight, which is consistent with the scheme we designed. The selection of approaching mode is unnecessary, and the flight track has only one turn direction, which simplifies the trajectory planning algorithm and guarantees the ease of calculation. In the S-turn phase, energy is consumed sharply in this method. Altitude and velocity are decreasing gradually in the latter phases. The inset of Figure 6 shows the boundary of S-turn phase where bank angle is $80^{\circ}$ (i.e., $\mu_{0}=80^{\circ}$ ). The width of the boundary guarantees that the vehicle arrives at the predetermined TAEM terminal point successfully. A few times of bank angle reversal can also be achieved. Figure 7 plots the 3D trajectory which shows the results as we expected.

In cases studies, the vehicle altitude and velocity are modified-both increase and decrease in altitude and velocity. Three runs are shown in Figures 8 and 9: case 1, nominal 


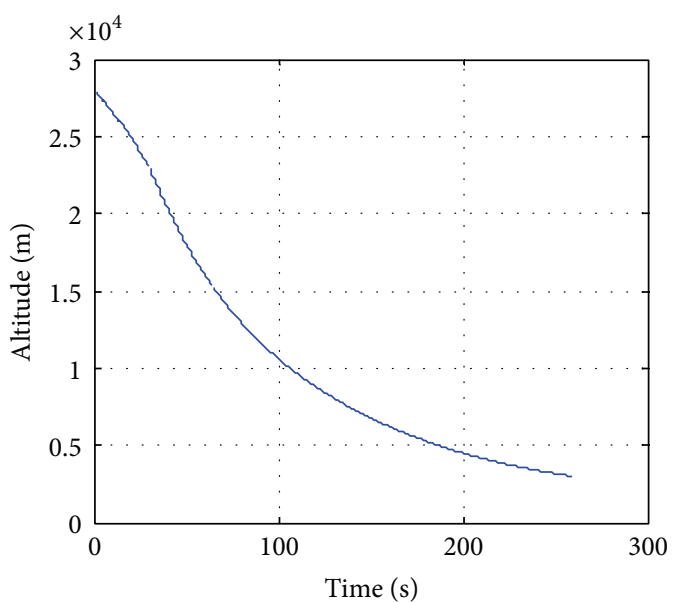

(a)

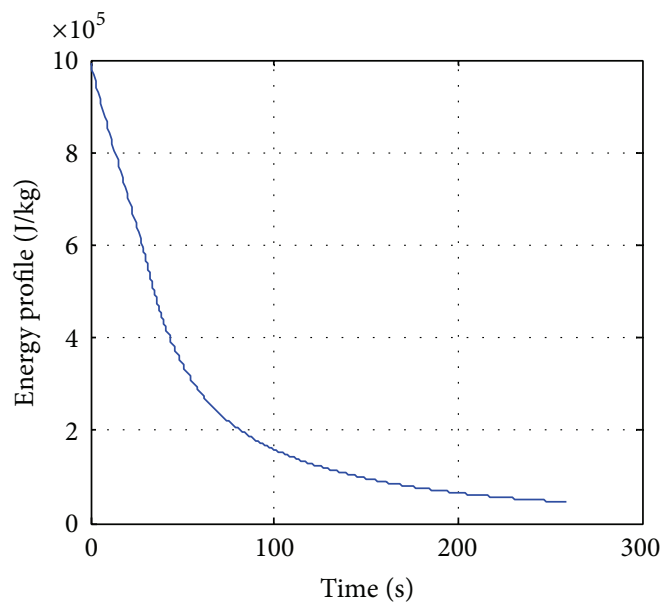

(c)

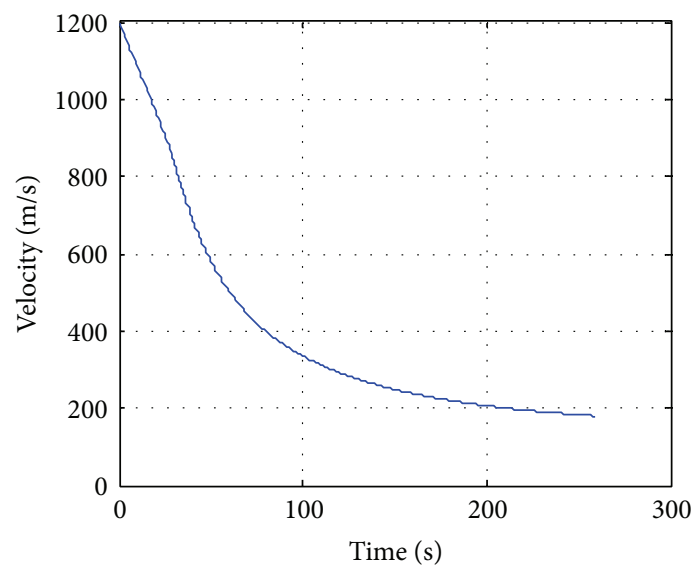

(b)

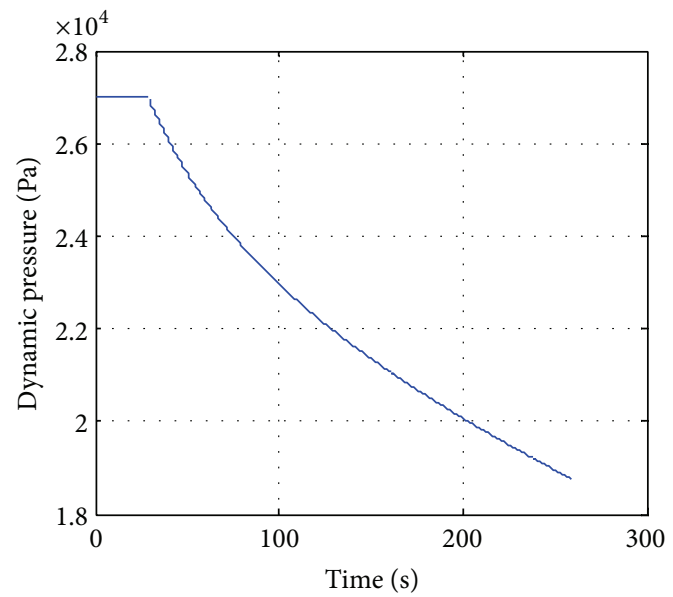

(d)

FIGURE 5: Altitude, velocity, energy, and dynamic pressure profile for TAEM trajectory.

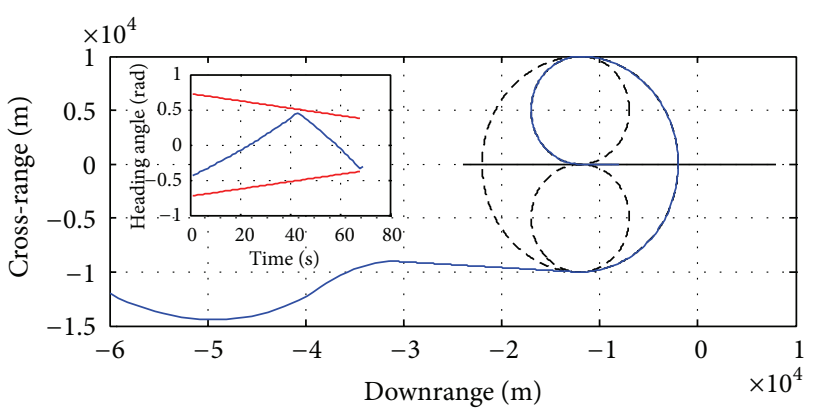

FIGURE 6: TAEM ground track trajectory.

altitude; case 2, $3 \mathrm{~km}$ increase in altitude; case 3, $3 \mathrm{~km}$ decrease in altitude. For these cases, Figure 8 plots their altitude profiles and Figure 9 plots the ground track trajectories. Although the altitude profiles show different initial points in different runs, they reach the same altitude at the end of TAEM. From the ground track plots, it can be seen that the higher energy condition is, the longer ground track resulted to dissipate energy, and the lower energy condition is, the shorter ground track resulted to preserve energy. Three runs are shown in Figures 10 and 11: case 1, nominal velocity; case $2,100 \mathrm{~m} / \mathrm{s}$ increase in velocity; case $3,100 \mathrm{~m} / \mathrm{s}$ decrease in velocity. Figure 10 plots the velocity profiles and Figure 11 plots the ground track trajectories. Although the velocity profiles show different initial points in different runs, they reach the same velocity at the end of TAEM. The higher energy condition is, the longer ground track resulted to dissipate energy, and the lower energy condition is, the shorter ground track resulted to preserve energy.

\section{Conclusion}

A new guidance scheme based on energy-to-range ratio (E-R) has been developed for the terminal area energy management (TAEM) phase of RLV. The guidance method employs a trajectory planning algorithm which takes no account of approaching mode with only one turn heading and generates the reference path expediently based on 


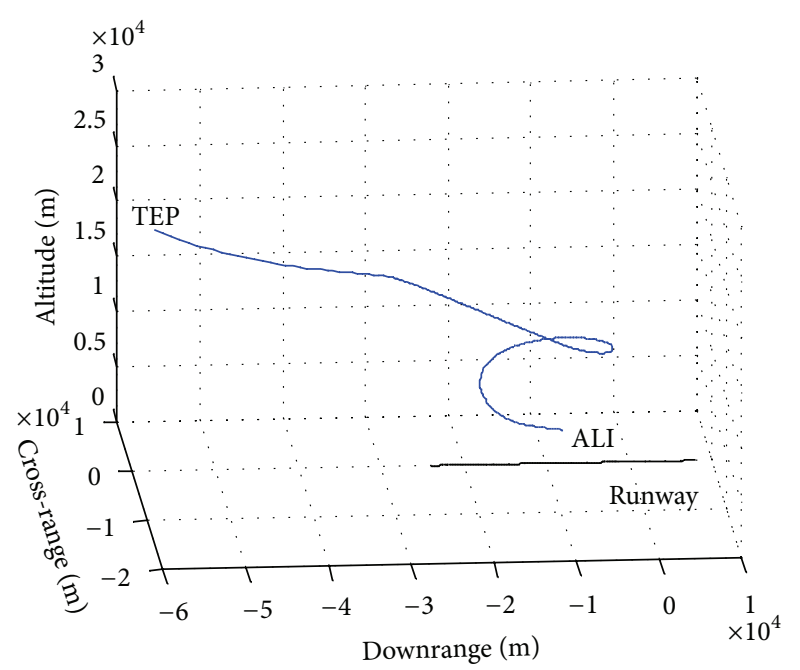

Figure 7: Three-dimensional TAEM trajectory.

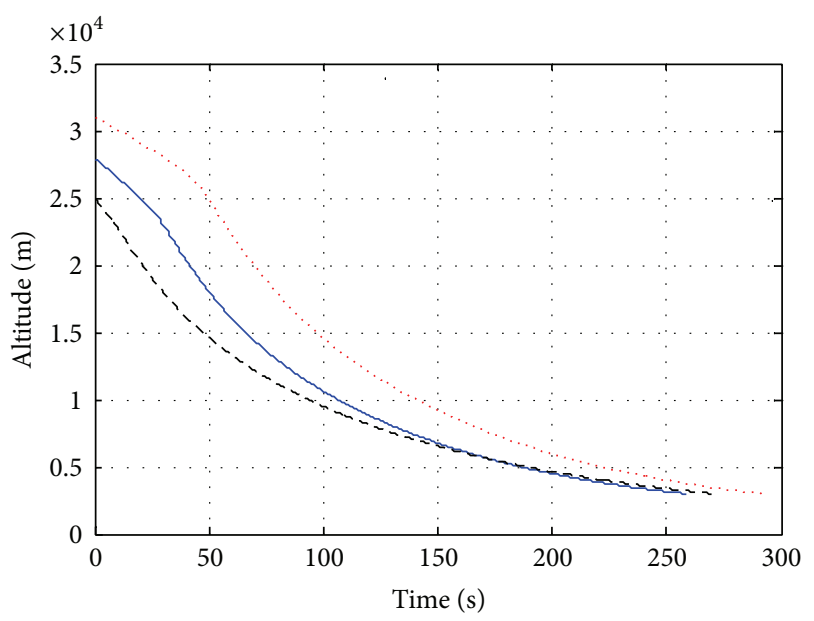

- Nominal

$3 \mathrm{~km}$ altitude increase

- - $3 \mathrm{~km}$ altitude decrease

FIgURE 8: Altitude profile for different energies.

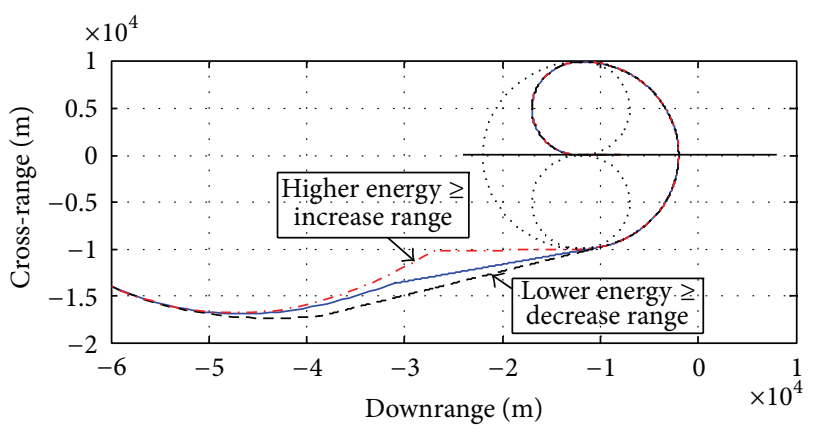

FIGURE 9: TAEM ground track trajectories for different altitudes.

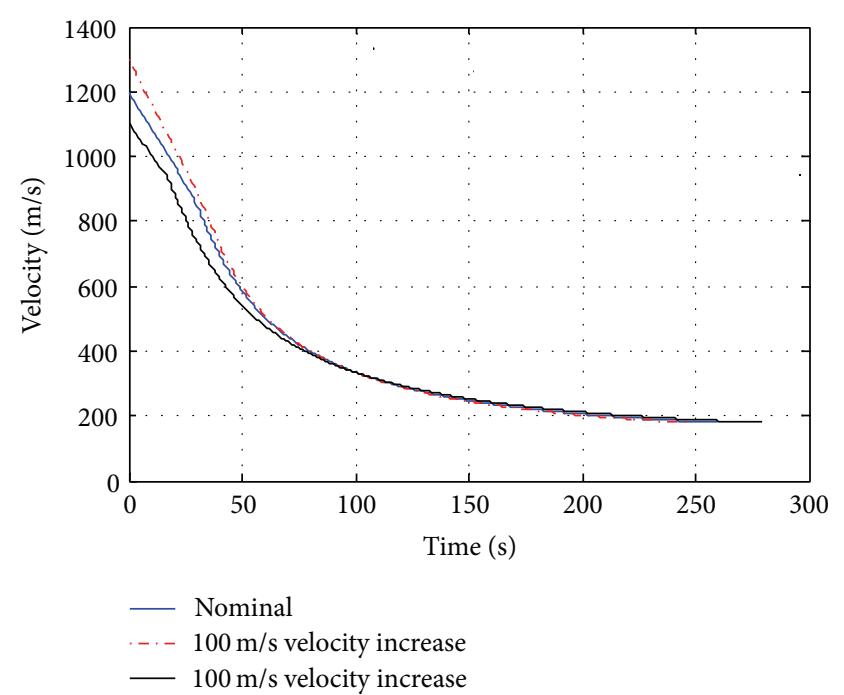

Figure 10: Velocity profile for different energies.

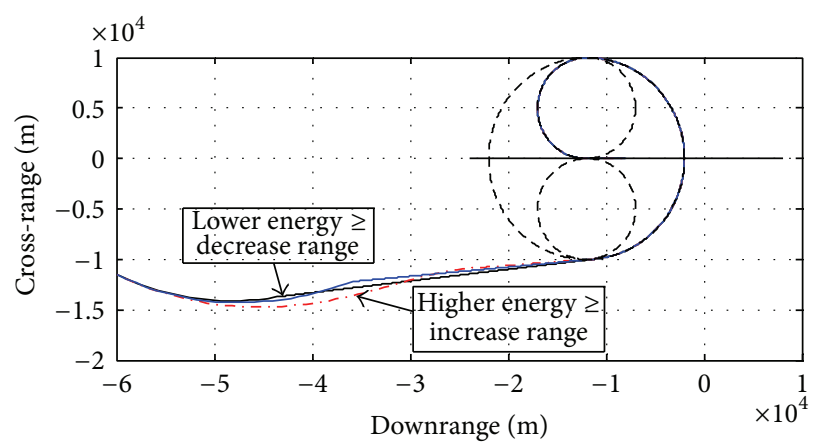

FIGURE 11: TAEM ground track trajectories for different velocities.

the predicted ground track, the energy state, and the vehicle's aerodynamic performance.

Several simulations were conducted in order to demonstrate the proposed guidance algorithm. Scenarios involving initial energy variations, heading dispersions, pneumatic coefficient deviations, and air density deviations were simulated, although not shown totally. The results show that the trajectory planning guidance method successfully targeted the desired ALI state and satisfied intermediate constraints for all initial surplus energy cases. Furthermore, many of the extreme cases required significant changes in the TAEM reference profile, such as large changes in the HAC position and final HAC radius. These changes seem to be necessary in order to satisfactorily accomplish the ALI conditions while at the same time satisfying the intermediate path constraints. Based on these analyses, the trajectory planning guidance algorithm is a viable candidate for engineering implementation in order to improve reliability and safety of future reusable launchvehicles. 
In future work, we will further improve the performance of the trajectory planning algorithm via some evolutionary algorithms, such as fuzzy control methods [16-18] and neuronal networks control methods $[19,20]$. It is important to optimize our results of guidance algorithm by utilizing some evolutionary methods.

\section{Conflict of Interests}

The authors declare that there is no conflict of interests regarding the publication of this paper.

\section{Acknowledgment}

The research work presented in this paper was supported by the Fundamental Scientific Research Project of Beihang University under Grant no. YWF-14-YQGD-007.

\section{References}

[1] J. C. Harpold and C. A. Grave, "Shuttle entry guidance," NASA n79-74791, NASA, 1979.

[2] T. E. Moore, "Space shuttle entry terminal area energy management," NASA Technical Memorandum 104744, NASA, 1991.

[3] Z. Shen and P. Lu, "Onboard generation of three-dimensional constrained entry trajectories," Journal of Guidance, Control, and Dynamics, vol. 27, no. 1, pp. 111-121, 2003.

[4] J. P. Saldia, A. Cimino, W. Schulz, S. Elaskar, and A. Casta, "Atmospheric reentry dynamics of conic objects," Mathematical Problems in Engineering, vol. 2009, Article ID 859678, 14 pages, 2009.

[5] K. R. Horneman and C. A. Kluever, "Terminal area energy management trajectory planning for an unpowered reusable launch vehicle," AIAA Paper 2004-5183, AIAA, 2004.

[6] C. A. Kluever, K. R. Horneman, and J. D. Schierman, "Rapid terminal-trajectory planner for an unpowered reusable launch vehicle," AIAA Paper 2009-5766, AIAA, 2009.

[7] N. R. S. Filipe, E. Weedrt, E. Kampen, Q. P. Chu, and J. A. Mulder, "Terminal area energy management trajectory optimization using interval analysis," AIAA Paper 2009-5768, AIAA, 2009.

[8] D. Abbasi and M. Mortazavi, "A new concept for atmospheric reentry optimal guidance: an inverse problem inspired approach," Mathematical Problems in Engineering, vol. 2013, Article ID 419409, 13 pages, 2013.

[9] J. M. Hanson, "A plan for advanced guidance and control technology for 2nd generation reusable launch vehicles," AIAA Paper 02-4557, AIAA, 2002.

[10] J. M. Hanson, "New guidance for new launchers," Aerospace America, vol. 41, no. 3, pp. 36-41, 2003.

[11] J. M. Hanson and R. E. Jones, "Test results for entry guidance methods for space vehicles," Journal of Guidance, Control, and Dynamics, vol. 27, no. 6, pp. 960-966, 2004.

[12] C. A. Kluever, "Terminal guidance for an unpowered reusable launch vehicle with bank constraints," Journal of Guidance, Control, and Dynamics, vol. 30, no. 1, pp. 162-168, 2007.

[13] B. T. Burchett, "Fuzzy logic trajectory design and guidance for terminal area energy management," Journal of Spacecraft and Rockets, vol. 41, no. 3, pp. 444-450, 2004.
[14] K. Grantham, "Adaptive critic neural network based terminal area energy management/entry guidance," AIAA Paper 20030305, AIAA, 2003.

[15] C. E. Hall and Y. B. Shtessel, "Sliding mode disturbance observer-based control for a reusable launch vehicle," Journal of Guidance, Control, and Dynamics, vol. 29, no. 6, pp. 1315-1328, 2006.

[16] H. Li, X. Jing, and H. K. Lam, "Fuzzy sampled-data control for uncertain vehicle suspension systems," IEEE Transactions on Cybernetics, 2013.

[17] H. Li, X. Jing, and H. R. Karimi, "Output-feedback-based $H_{\infty}$ control for vehicle suspension systems with control delay," IEEE Transaction on Industrial Electronics, vol. 61, no. 1, pp. 436-446, 2014.

[18] H. Li, J. Yu, and C. Hilton, "Adaptive sliding mode control for nonlinear active suspension vehicle systems using T-S fuzzy approach," IEEE Transaction on Industrial Electronics, vol. 60, no. 8, pp. 3328-3338, 2013.

[19] Y. Tang, H. Gao, and J. Kurths, "Multiobjective identification of controlling areas in neuronal networks," IEEE/ACM Transactions on Computational Biology and Bioinformatics, vol. 10, no. 3, pp. 708-720, 2013.

[20] Y. Tang, H. Gao, and J. Kurths, "Evolutionary pinning control and its application in UAV coordination," IEEE Transactions on Industrial Informatics, vol. 8, no. 4, pp. 828-838, 2012. 


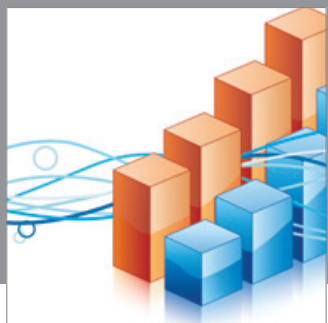

Advances in

Operations Research

mansans

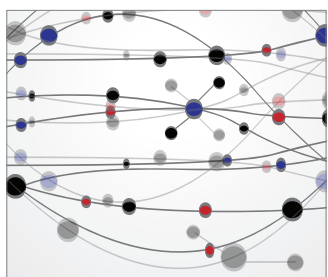

The Scientific World Journal
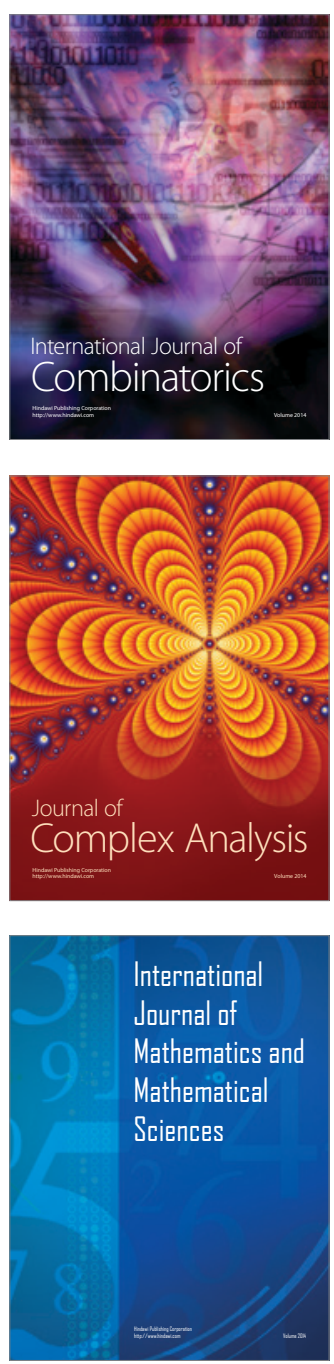
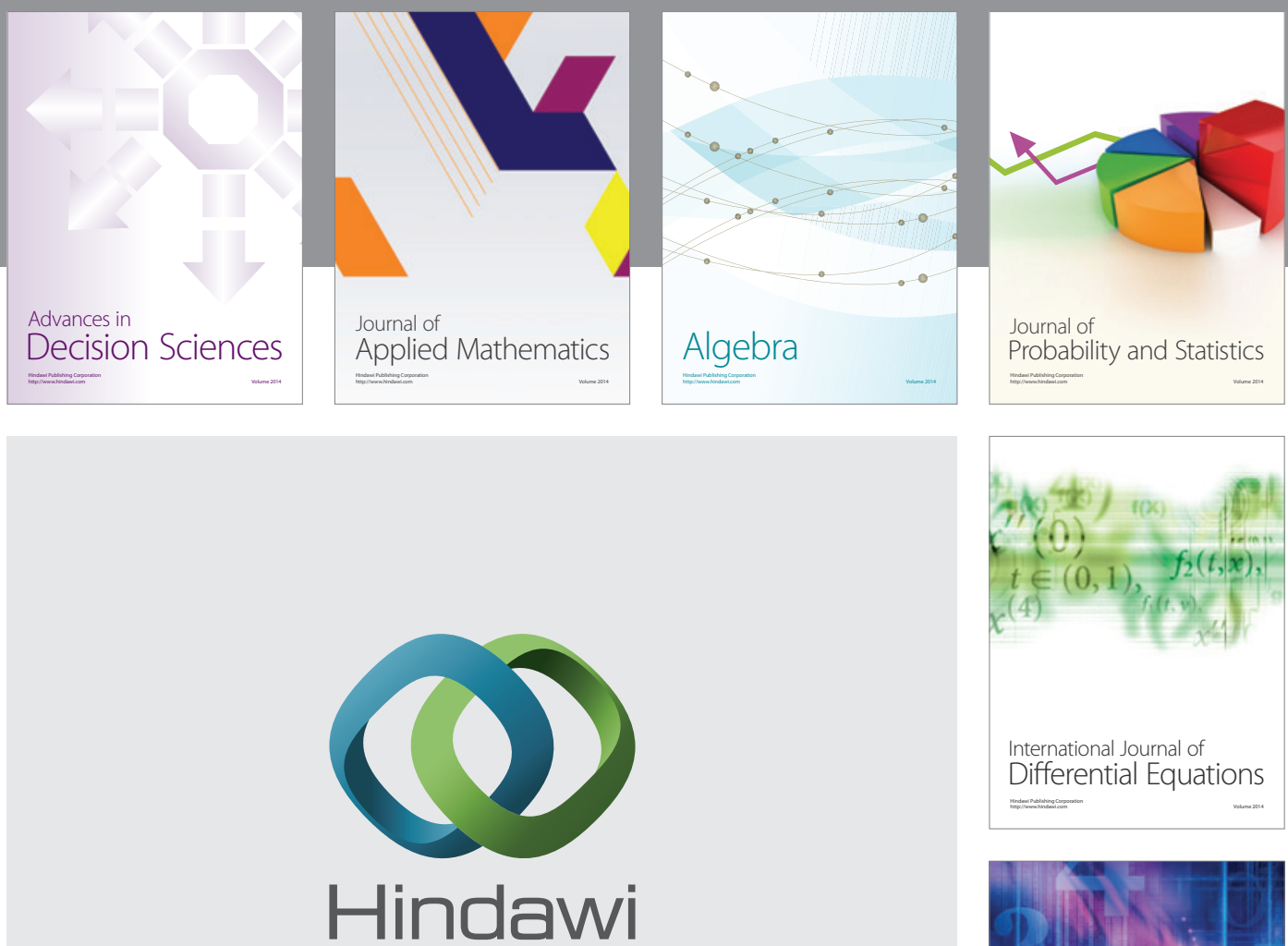

Submit your manuscripts at http://www.hindawi.com
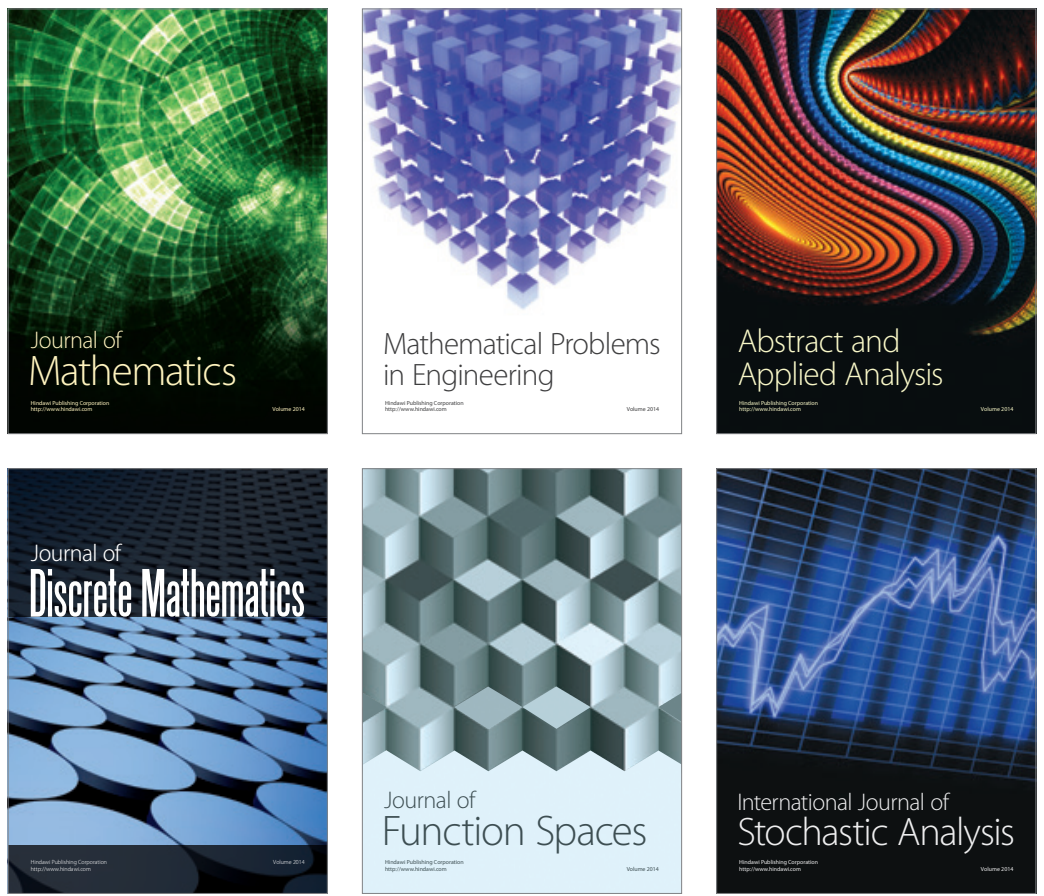

Journal of

Function Spaces

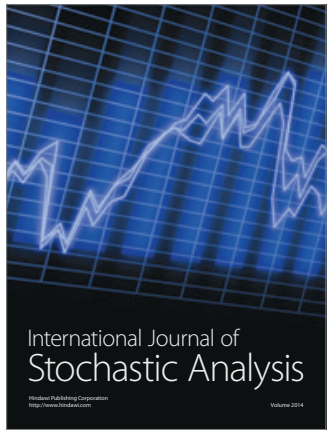

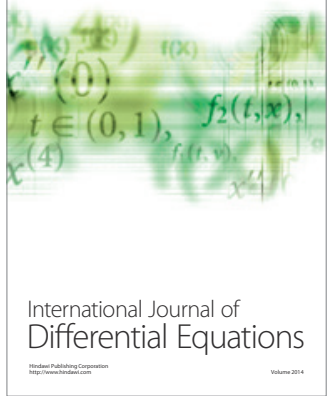
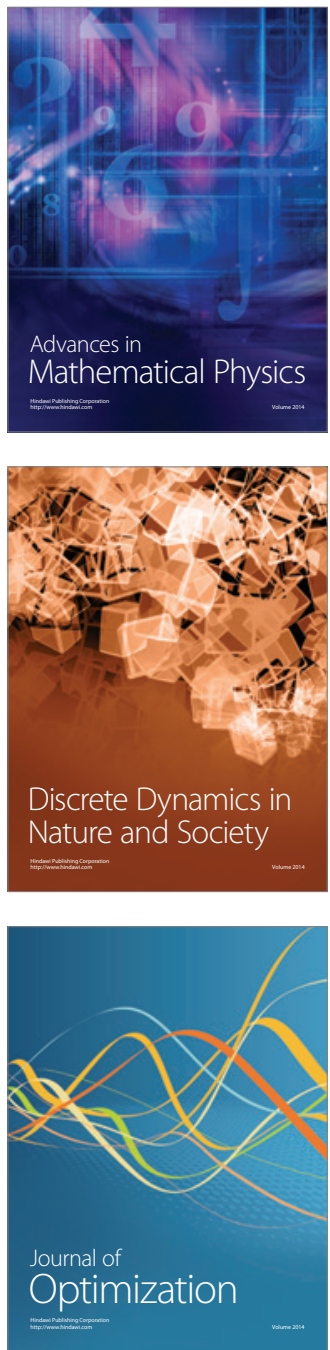